\title{
Recognising railway infrastructure elements in videos and drawings using neural networks
}

Simon Vilgertshofer MSC

Research Associate, Chair of Computational Modeling and Simulation,

Technical University of Munich, Munich, Germany (Orcid:0000-0003-42712076) (corresponding author: simon.vilgertshofer@tum.de)

\section{Deian Stoitchkov BSC}

MSc student, Chair of Computational Modeling and Simulation, Technical University of Munich, Munich, Germany
André Borrmann Dr.-Ing.

Professor, Chair of Computational Modeling and Simulation, Technical University of Munich, Munich, Germany (Orcid:0000-0003-2088-7254)

Alexander Menter MSc

Software Engineer, Signon Deutschland GmbH, Munich, Germany

Cengiz Genc Dipl. Inf. (FH)

Division Manager Software, Signon Deutschland GmbH, Munich, Germany

Accurate data in the form of technical drawings of built assets are an essential requirement for the successful operation and reconstruction of the built environment. When the consistency between these data and the real-world situation cannot be ensured, the data are not reliable and need to be verified by comparing drawings and reality. Depending on the size and the number of assets, this may involve an enormous amount of manual effort. In this paper, an approach to supporting and automating this process by utilising machine learning concepts has been developed in the context of railway engineering. The research focuses on two aspects: the analysis of technical drawings to locate plan symbols and the recognition of infrastructure elements in video data of railway lines. Both tasks are time-intensive and errorprone processes when done manually. In this paper, it is described how the capabilities of convolutional neural networks are employed in analysing images from video data and of technical drawings, in order to detect automatically the location of railway infrastructure elements. The outcome of these two approaches can then be compared with catalogue elements and to check the consistency of corresponding technical drawings.

\section{Introduction}

The railway network in Germany consists of more than $30000 \mathrm{~km}$ of railway lines. Because this integral part of German infrastructure has grown gradually over the past century, the railway lines and equipment are of varying technological levels. The same is true of the corresponding technical drawings. However, the operation, maintenance, remediation and refurbishment and even the reconstruction of railway infrastructure rely heavily on the availability of such technical drawings, which need to be exact and up to date. In a vast railway network of several thousand kilometres that has expanded over the course of many decades, discrepancies between archived drawings and the built infrastructure are almost inevitable, due to a variety of reasons. As the process of comparing and updating technical drawings involves an enormous amount of manual effort, an approach was developed for supporting and automating parts of this process, by utilising concepts developed in the field of machine learning.

The research presented here is part of the RIMcomb research project (Railway Information Modeling: Equipment Technology for Rail Infrastructures (Stoitchkov et al., 2019; Vilgertshofer et al., 2018)). Among the main goals of the research project is the digitisation of the information from conventional two-dimensional drawings depicting railway equipment. While many technical drawings of German railway infrastructure are now available as digital images, their interpretation and, most importantly, the understanding of the various symbols that they contain has to be undertaken manually to create a semantically rich digital twin.

The presented approach aims to support this process in order to reduce the manual effort, by automating at least parts of this image-interpretation process. The first step towards this goal is the automatic recognition and highlighting of symbols on a given drawing and the subsequent extraction of their quantity and location. To achieve this, convolutional neural networks (CNNs) were employed, as this method showed promising results in a former study (Stoitchkov, 2018) and as they have been applied successfully in image recognition for a large number of applications. In this approach, a process was developed to train these networks without the need for a time-consuming manual labelling process to generate training and testing data. The results can then be compared with the actual situation on the corresponding railway track in order to check for inconsistencies or to confirm the accuracy of a drawing.

Naturally, this comparison process also requires the recognition of infrastructure elements on a railway track in the real world as a counterpart. In the scope of the RIMcomb research project, an approach to automating this process based on object detection in videos captured from railway tracks was developed (Genc et al., 2018). The further refinement of this approach is described in this paper. A combination of both approaches has been implemented that enables the semi-automated execution of the otherwise highly labour-intensive comparison of the as-planned and as-built situation of railway infrastructure assets (Figure 1).

This paper is structured as follows. In Section 2, existing approaches to detecting and classifying symbols in technical drawings and video data are discussed. Furthermore, the technological background of the approaches is described. Section 3 addresses the automated recognition of infrastructure elements in video data, explaining the 


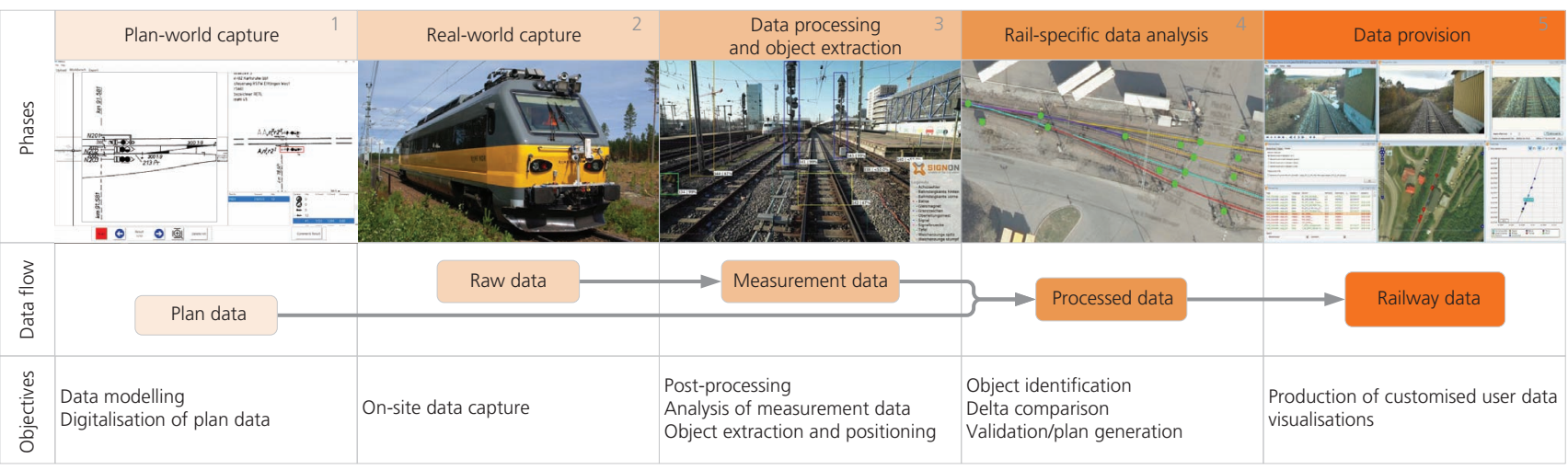

Figure 1. Overview of the overall workflow of the approach

method, implementation work and general quality of the results. Accordingly, Section 4 provides a detailed description of how symbols in technical drawings of railway tracks can be recognised automatically. The following section shows how the results of both parts (symbol detection in drawings and object detection in videos) of the approach are combined. Finally, the findings are summarised and discussed in the context of practical applicability, as are possible future research possibilities.

\section{Related research and theoretical background}

The following section gives an overview of other approaches to the automatic recognition of symbols in technical drawings and in the field of object detection in images and videos. They form the basis of development for the research presented in this paper. Additionally, Section 2.3 describes the main concepts in the field of machine learning - namely, artificial neural networks - that were used in the presented method. This related research is mainly introduced and referenced for suggested further reading and for putting this paper into a scientific context. It is, however, not described in complete detail, as this would be beyond the scope of this paper or would not further contribute to it.

\subsection{Symbol recognition in technical drawings}

Computer-automated symbol recognition in technical drawings is a well-known problem in the engineering field. In technical drawings, symbols often appear to be greatly distorted or overlapped with other objects, making their recognition a significant challenge. However, numerous solutions to this problem have already been proposed.

Luqman et al. (2009) describe a method that represents symbols by their graph-based signatures, as well as a Bayesian network (a certain type of probabilistic graphical model that represents the conditional dependencies between a set of variables as a graph) that is trained to encode the common probability distribution of symbol signatures. Another approach is described by Weber and Tabbone (2012), whereby the template-matching operator HMTAIO (Hit-or-miss Transform Adapted to Information Overlapping) is used. Hit-or-miss transform is a general binary operation that can be used to look for certain patterns of foreground and background pixels in an image. The advantage of the approach proposed in this work is its robustness against occlusion and overlapping. Another interesting approach to symbol spotting is proposed by Rusiñol and Lladós (2005). The authors describe their approach as follows: '[...] a method to determine which symbols are probable to be [sic] found in technical drawings using vectorial signatures. These signatures are formulated in terms of geometric and structural constraints between segments, as parallelisms, straight angles, etc.' (Rusiñol and Lladós, 2005: p. 35). Further research in this area was conducted by Rezvanifar et al. (2019) reviewing different methods of symbol recognition with a focus on architectural drawings. Similar research on architectural drawings was made by Rezvanifar et al. (2020), this time using deep learning and adapting an object detection framework based on the you-only-look-once architecture. Different symbol recognition methods are also described by Le Bodic et al. (2012), Nayef et al. (2012) and Ziran and Marinai (2018).

Although many approaches have been proposed, these have not been able to deliver satisfactory results for detecting railway equipment symbols. However, a concept that has been successfully applied to the challenge of recognising objects in images is the application of artificial neural networks (see Section 2.3). A preliminary study by Stoitchkov (2018) stated that using this method shows promising results and can be applied in various contexts. It not only is invariant to scale and rotation but also works for heavily distorted symbols and recognises many types of symbols, thus demonstrating its practical applicability.

\subsection{Recognition of infrastructure elements in video data}

Complementary to symbol recognition, the next step towards further automating the process of detecting and cataloguing infrastructure elements in railway networks is to record individual railway lines with cameras that are mounted on a train (see Figure 1). The recorded video data can then be analysed using a tracking-by-detection approach, which means that an object detection algorithm runs on individual frames, and the resulting detections of the same element across different frames are linked together to form object detection tracks. 
Smart Infrastructure and Construction

Volume 172 Issue 1
Recognising railway infrastructure

elements in videos and drawings using

neural networks

Vilgertshofer, Stoitchkov, Borrmann, Menter and Genc
According to Bochinski et al. (2017), a highly performant object detector can significantly increase the reliability of these object detection tracks. Thus, it is essential to implement an object detector that is able to both recognise as many objects as possible and locate them very precisely in an image. Benenson et al. (2014) evaluated a variety of object detection algorithms and concluded that finding features that suppress the differences in objects of the same category while highlighting the differences in objects of different categories is of crucial importance. Additionally, those features need to be scale, rotation and translation invariant as well as invariant to changes in illumination.

However, recognising objects in an image is only the first step. The main goal of object detection is to find the precise position of an object. One of the first detection algorithms in computer vision uses a sliding-window approach, where a rectangle moves over the image and the region of the image within that rectangle is classified (Papageorgiou and Poggio, 2000). As an exhaustive search over the image is computationally expensive, the aspect ratio of the rectangle is usually fixed. Based on this approach, Viola and Jones (2004) developed a face detector. However, according to Süße and Rodner (2014), this method does not perform well on objects of different classes.

Dollár et al. (2014) developed the aggregated channel feature detector, which uses image channels of different scales and a cascade of classifiers. As the object detectors by Viola and Jones (2004) and Dollár et al. (2014) also use sliding windows with fixed aspect ratios, these approaches are not very robust against strong deformations and high intraclass variance in shape. In order to overcome this problem, Felzenszwalb et al. (2010) developed a detector that does not detect an object as a whole, but represents an object as a combination of its parts using deformable part models. Girshick et al. (2014) presented an approach where features are learned using region-based CNNs (R-CNNs). Instead of searching for objects in the whole image as with sliding-window techniques, R-CNNs use a region proposal method, such as the Region Proposal Networks for Faster R-CNNs (Ren et al., 2015). Furthermore, a bounding-box regression algorithm refines the bounding boxes to enclose the object tightly. Many of the recent advances in visual recognition tasks are due to the development of deep neural networks based on the Faster R-CNN system as described in the paper by $\mathrm{He}$ et al. (2015). In order to track multiple objects in a video, the aforementioned tracking-by-detection approach has emerged as the preferred paradigm (Bergmann et al., 2019; Bochinski et al., 2018; Yoon et al., 2019). Here, an object detector analyses individual video frames to recognise a specific object. The object tracker then links corresponding detections in different frames across time (see Figure 2) and keeps the identity of the tracked objects. The linking step itself can be challenging due to the uncertainty about the number of objects to track and the uncertainty about when and where those objects enter and leave a scene. Furthermore, missing or false detections can cause a high number of identity switches (i.e. how many times an object changes its identity number across all the frames).

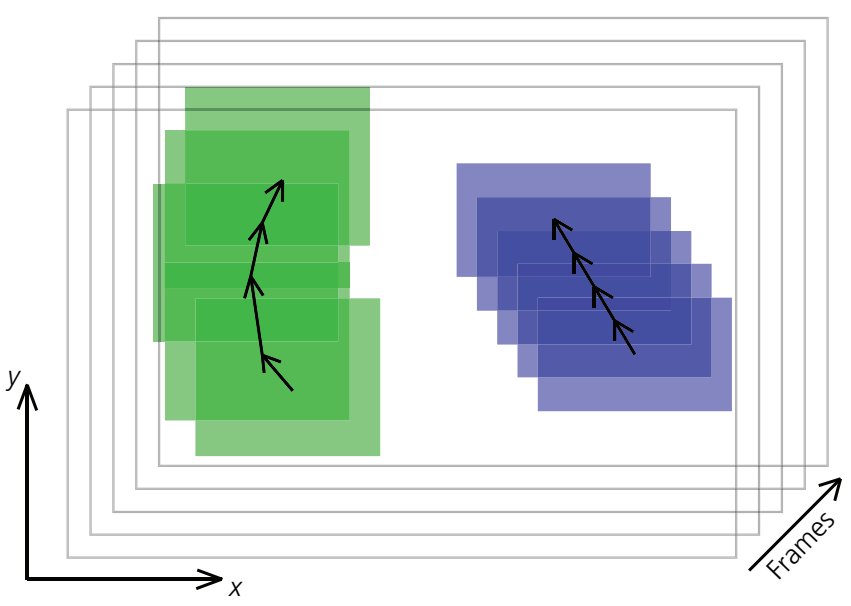

Figure 2. Accepted detections of two objects (green/blue rectangles) in different video frames are combined to form detection tracks by their spatial overlap between time steps (figure based on Bochinski et al. (2017))

Although object tracking has been an active research area in the past decades (Wu et al., 2013), increasingly complex tracking models achieved only marginally better results according to Bergmann et al. (2019). However, with high frame rates and very accurate object detectors, far simpler tracking algorithms performed as well as or even outperformed the state-of-the-art algorithms on public object detection and tracking data sets. Tracking algorithms in various approaches (Bergmann et al., 2019; Bewley et al., 2016; Bochinski et al., 2017, 2018; Wojke et al., 2017) link detections with a high overlap in consecutive frames together to individual detection tracks. As those simple approaches rely heavily on the output of the object detector, the focus of the RIMcomb research project was primarily on developing a highly accurate object detector.

\subsection{Convolutional neural networks}

Artificial neural networks are used for different tasks such as text, speech and image recognition and computer vision. A simple example of a neural network is shown in Figure 3. By adjusting

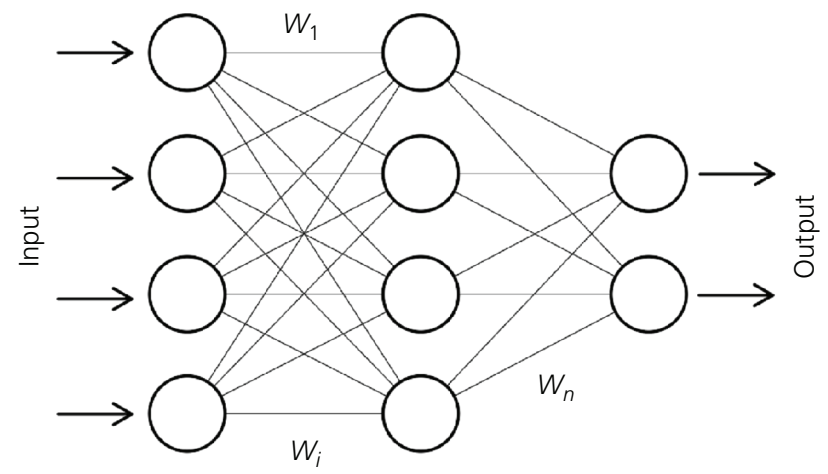

Figure 3. Simplified neural network structure 
Smart Infrastructure and Construction

Volume 172 Issue 1
Recognising railway infrastructure

elements in videos and drawings using

neural networks

Vilgertshofer, Stoitchkov, Borrmann, Menter and Genc the weights (marked with a $W$ in Figure 3 ) between the neurons, an output is calculated from a given input. In the present case, the input is the feature map, which has been previously calculated. The output is an array with the coordinates of the symbol that is to be recognised.

CNNs have been used in the approaches presented in this paper, as they have been applied successfully in image recognition for a large number of applications. The name 'convolutional neural network' originates from the mathematical operation called convolution (a special type of linear operation) used by the network. Therefore, CNNs are just neural networks that have at least one layer in which a convolution is used in place of general matrix multiplication. One of the first CNNs developed is the LeNet (LeCun et al., 1998), and since its creation, there has been a tremendous improvement in the field of image recognition and object recognition in general. CNNs use filters to detect patterns (or features) in an image. An image is analysed to find these patterns, and a value is stored that represents how good each pattern matches the image at specific locations. In this way, an image can be inspected for features such as horizontal or vertical lines. By way of combination, they can represent more complex shapes such as symbols or objects. Figure 4 shows an example for two filters of a horizontal and a vertical line on the left side and how they are matched in the image on the right-hand side.

The actual filtering is made by sliding the filter over a bigger image and comparing it against a small patch (the red, blue and green boxes in Figure 4) from the large image, whereby the patch always has the same size as the filter. Each filter pixel is multiplied with the corresponding pixel from the image, and the resulting values are added up. Afterwards, this value is divided by the pixel number of the filter. For example, if the feature that represents a vertical line (upper filter in Figure 4) is compared with the part of the image that is marked by a red rectangle in Figure 4, the result would be 3 . There are six pixels with the same value $(-1)$ and three pixels that have different values $(-1$ and 1$)$.
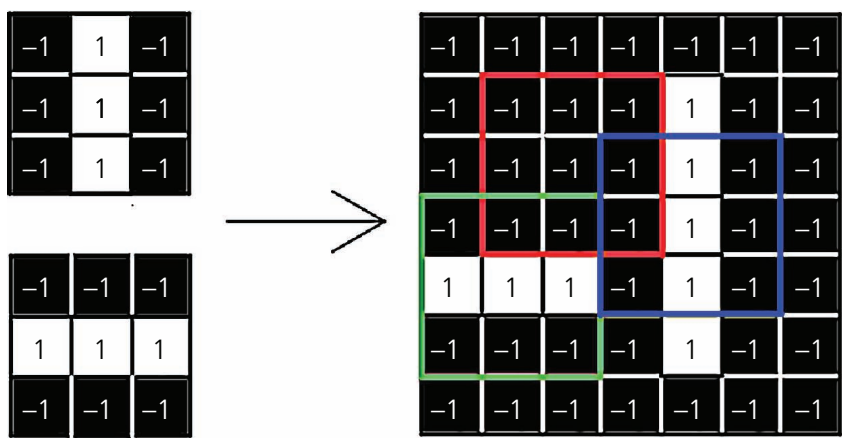

Figure 4. Searching for filters in an image consisting only of black and white pixels. On the left are two different filters with a size of $3 \times 3$ pixels. On the right is an example image with a size of $7 \times 7$ pixels. The blue, red and green boxes represent certain patches of the image that are compared with the filters
All the pixels with the same values are added together; the rest is subtracted from the sum, resulting in the number 3 . This result is then divided by the number of pixels in the feature, which in this case is 9 . For the given example above, the final result would be 0.33 ( 3 divided by 9 ). In the case of binary images, if the filter is found in the image with a $100 \%$ accuracy, the result for this location of the image is 1 (upper filter with the blue box in the image). On the other hand, if the filter has no shared values with the current location in the image, the result would be -1 . The overall result is a map that represents where the different features occur in the image. By matching the feature for every possible location in the image, a convolution is made, hence the name 'convolutional neural networks'. A rectified linear unit (ReLU) activation function can then be used to normalise the values in the feature map that was calculated, which means that all values that are below zero are amended to zero (Figure 5). This step simplifies the calculation of the neural network. ReLU is defined as $f(x)=\max (0, x)$.

Images usually consist of thousands or even millions of pixels, which can make the training of a CNN computationally expensive, as larger images result in a larger feature map. Therefore, an operation is introduced that reduces the size of the feature map. Here, the so-called max pooling operation is used. It takes only the maximum value of a given window of a certain size (pool size) and saves it at the correct location in a new, smaller feature map (Figure 6). These techniques can be used multiple times in layers, whereby each layer takes as its input the output of the previous layer. The image becomes more filtered after each convolution layer and smaller after each pooling layer

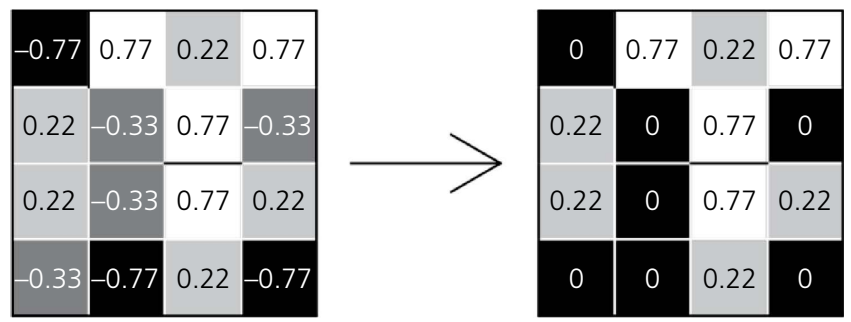

Figure 5. A feature map transformed with ReLU

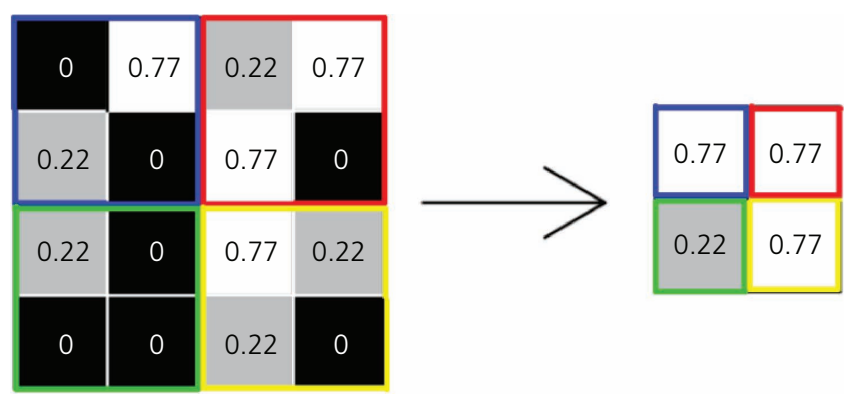

Figure 6. Max pooling with a pool size of $2 \times 2$ pixels 
Smart Infrastructure and Construction Volume 172 Issue 1
Recognising railway infrastructure

elements in videos and drawings using

neural networks

Vilgertshofer, Stoitchkov, Borrmann, Menter and Genc
(Stoitchkov, 2018). The extracted features are then processed further by the neural network.

In the training process, the weights of the network are systematically adjusted to minimise the difference between the output that it has generated and the ground truth result that is targeted. This is achieved by adjusting the weights for every connection until the desired result is achieved. If the generated output differs from the desired result, the weights are adjusted. One pass over all images with multiple adjustments of the weights is called an epoch. When the calculated result is far from the required result, the weights are greatly modified. If the results are close, the weights are adjusted only slightly. This is performed multiple times, and the error between the real and generated results is calculated on each occasion. The smaller the error, the better that the neural network is trained. In order to reach a good level of accuracy (i.e. exact recognition), thousands of training images and many epochs are necessary.

However, one of the biggest challenges in training a neural network is the prevention of overfitting. Overfitting occurs when a predictive model is too dependent on the specific examples of the data that it is trained with and cannot generalise well to unknown data. For example, this would occur if the network is trained on only one instance of a very specific symbol for a signal. In this case, the network would not recognise a symbol that still represents the same element but differs very slightly from the symbol that was used for training. This problem can be solved to some extent by using a dropout layer. A dropout layer randomly turns off some of the neurons in the neural network (circles in Figure 3), which prevents adaptation to specific images. This matter is discussed in detail in Section 4.2.1.

\section{Recognition of infrastructure elements in video data}

\subsection{Methodical approach}

One part of the presented approach (see columns 2 and 3 in Figure 1) to digitising railway infrastructure drawings involved the recording of geo-referenced video data. As individual measurement runs can be up to $10 \mathrm{~h}$ long, detecting infrastructure elements automatically in images and videos using machine learning is an essential component. The objective of this part of the presented approach was to detect the following elements: balise, PZB inductor, signal, signal gantry, railroad switch, panel, axle counter, railway platform and fouling point. Examples of those elements can be seen in a video still given in Figure 7.

In order to solve this task, the procedure was broken into four main parts: data acquisition, machine learning and object detection, post-processing techniques and evaluation.

\subsubsection{Data acquisition}

Although there has been much progress made in terms of object detection algorithms, one challenge remaining is the amount of data that are needed to train a predictive model. While there are

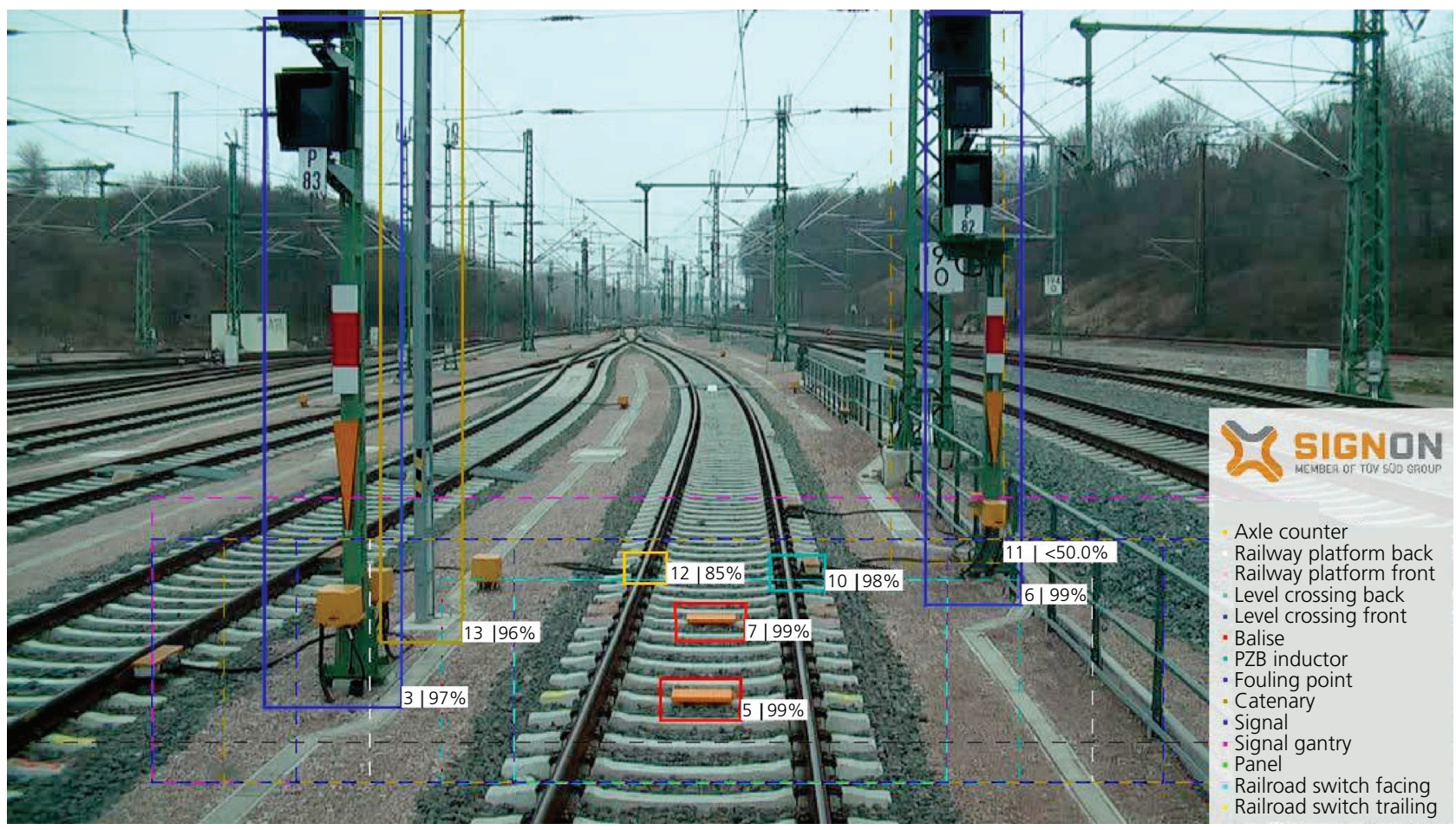

Figure 7. One video frame with the bounding boxes of the detected objects and the detection score 
Smart Infrastructure and Construction Volume 172 Issue 1
Recognising railway infrastructure

elements in videos and drawings using

neural networks

Vilgertshofer, Stoitchkov, Borrmann, Menter and Genc plenty of open-source data sets for object classes such as vehicles or pedestrians, to the best of the authors' knowledge, there is no such data set for railway infrastructure elements.

However, as Signon Deutschland $\mathrm{GmbH}$ (an engineering company participating in the RIMcomb research project) has performed on-site infrastructure video captures for many years (see Figure 8), hundreds of hours of previously captured video data from different countries were available for the presented approach. These data were collected for manual element recognition, and therefore, every video has a corresponding database in which each real-world infrastructure element is annotated, among other things, with a video frame number. Hence, individual frames containing objects of interest can be extracted automatically, but the objects in those extracted frames still have to be annotated with bounding boxes by hand. Additionally, each frame is tagged with meta information, such as country, weather condition or year of recording.

\subsubsection{Machine learning and object detection}

The approach presented here uses supervised machine learning to train a predictive model, using the annotated image data as input. To this end, the data set is split into three sets: the training set, the development set and the test set. The training set is used to train the model, and the development set is used to evaluate the model on individual frames while the model is being trained. As the predictive model is optimised based on the evaluation of the development set, the test set is used to ensure that the predictive model is not only performing well on the development set. Furthermore, as the goal is to catalogue objects from georeferenced video scenes and not to detect objects in single images only, the test set is based on video sequences and will be discussed in the evaluation part of this section.

In order to train the model, a pretrained deep neural network based on Faster R-CNN (Ren et al., 2015) is used. Pretrained networks have been trained on millions of images by the community and have learned to classify images into 1000 object classes. By retraining the final layers of the network architecture with the new data, the pretrained network was fine-tuned using transfer learning to detect railway infrastructure elements as - according to Talukdar et al. (2018) - this process is supposed to improve the performance of object detection. Moreover, using a pretrained network is usually faster than training a neural network from scratch.

\subsubsection{Post-processing techniques}

Given that the object detection model is available, there are several further steps necessary to catalogue infrastructure elements using geo-referenced video data. A simple way to reduce false detections is to determine a region of interest (ROI) and to accept detections

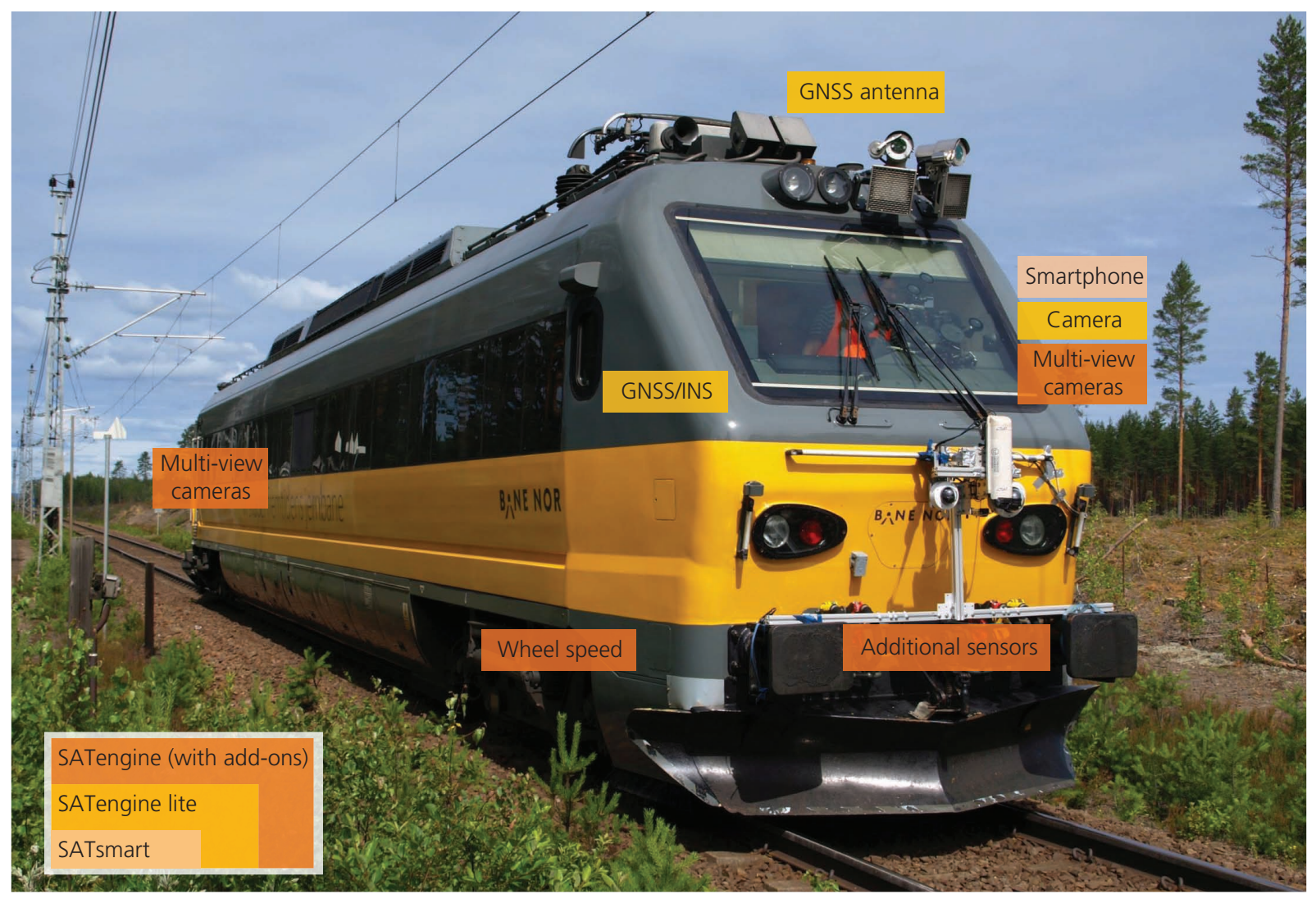

Figure 8. Set-up of the system for recording video data of railway lines. GNSS, global navigation satellite system; INS, inertial navigation system 
only in this area. However, in order to detect only elements that are close to the camera, the ROI is usually only in the lower half of the image and a detection is accepted when its bounding box has an intersection with the ROI (see Figure 7). Another simple method for reducing false detections is to determine minimum object width and height. False detections can be reduced by rejecting detections with a low score, although this may also lead to missed detections. Hence, a trade-off between risking missed detections and obtaining false detections must be made.

A real-world element is usually visible for $1-2 \mathrm{~s}$ or $25-50$ frames in the video and will therefore be detected multiple times. In the next step, the accepted detections are combined to form detection tracks (see Figure 2) with an identity, and those detection tracks can be filtered by determining a minimum length. Setting the length threshold too high will entail the risk of rejecting detection tracks for correct detections, while a low value means risking too many false detection tracks.

\subsubsection{Evaluation}

As already mentioned, video sequences are tested using object detection models in combination with the post-processing techniques described earlier. In order to catalogue the infrastructure elements, the result of the video analysis must be that each real-world element corresponds to one and only one detection track. In the case of the recognition of multiple detection tracks for the same object, only one detection track is considered correct, while the others are ignored. On the other hand, a detection track is considered correct even if it does not detect an object in every individual frame.

\subsection{Implementation and results}

The choice of the object detection architecture in this part of the RIMcomb project was based on the paper of Huang et al. (2016), where various modern convolutional object detection systems were evaluated for speed, memory and accuracy. The open-source machine learning framework TensorFlow (Abadi et al., 2016) offers pretrained models for those object detection systems that can be easily fine-tuned using the TensorFlow Object Detection application programming interface (Huang et al., 2016). Because - for the RIMcomb project - accuracy is more critical than speed, the Faster R-CNN Inception V2 (Szegedy et al., 2015) and Faster R-CNN ResNet50 (He et al., 2015) models turned out to have the best speed-accuracy balance for the purpose of the project.

In order to fine-tune the pretrained models, Signon Deutschland $\mathrm{GmbH}$ provided 50 video sequences that are split into 40 sequences for training, four for evaluation and six for testing $(80 \%$ for training and $20 \%$ for evaluating and testing). With a frame rate of 25 frames per second and an average video duration of around $2 \mathrm{~h}$, this corresponds to over 9 million individual frames. As not all of these frames contain railway infrastructure elements, those with elements of interest can be extracted automatically using the Signon Deutschland $\mathrm{GmbH}$ database, as mentioned in the preceding section. By extracting at least three frames for each real-world element, this corresponds to over 110000 bounding boxes in the training set, over 8500 in the evaluation set and over 9000 in the test set. In the early phase of this project, the objects were annotated entirely by hand. However, once the first fine-tuned models are available, the labelling process is performed automatically by the object detection model. In order to guarantee that the labels are correct, they are checked by two people.

With the image and the corresponding annotation files available, they were fed into the Inception V2 or ResNet50 neural network to adjust the model to detect railway infrastructure elements. While training the detection model, the accuracy of the model is assessed continuously with a weighted average of precision and recall $\left(F_{2}\right.$ score) using the training data, to determine the bias error, and using the development data to determine the variance error (error on the data that the model is not trained with). The bias error is essential for detecting systematic errors. If the difference between the bias and the variance error is too high, it is an indicator that the model is overfitted on the training data and cannot be generalised well to unknown data. However, the checkpoint with the lowest variance error is selected to export the predictive model. The exported object detection model is then tested on the test data set. The assessment is based on tracks of detections in video sequences, and only infrastructure elements that are related to the current railway track of the train are evaluated. The detection results of the test set when combining the object detection model with an object-tracking algorithm, a motion-estimation model and the post-processing techniques mentioned earlier can be seen in Table 1. Figure 7 shows one video frame with the bounding boxes of the detected objects.

\section{Symbol recognition in technical drawings}

\subsection{Methodical approach}

The second part of the presented approach (Neuronal Network Detection and Interpretation of Plan Symbols - NNdips) aims to facilitate the detection of symbols for large numbers of technical drawings - that is, drawings of railway lines. The process is composed of three main parts - namely, the import and preprocessing of drawings, the training of $\mathrm{CNNs}$ and the detection of symbols using the CNNs. All steps have been automated and thus provide a technique that does not require the manual generation of training data by users. An extensive data set of real technical drawings of railway lines formed the basis of developing and validating the approach. Since artificially generated data were used for training and testing (as explained in the following subsection), this is particularly important since the real technical drawings were used for validation of the results - that is, the network was used to detect symbols on these drawings - and the results were compared with the results of a manual detection process by a human in order to verify the capabilities of the CNN.

As there are numerous ways to utilise neural networks, it was first decided which type of neural network was to be used and how it should be applied to the data. Considering that the aim was to detect symbols in railway infrastructure drawings - usually, image files $\mathrm{CNNs}$ were chosen. As mentioned before, $\mathrm{CNNs}$ are particularly 
Table 1. Detection tracks and error rates for the recorded railway track (as of May 2019)

\begin{tabular}{|c|c|c|c|c|c|c|}
\hline \multirow{2}{*}{ Element } & \multirow{2}{*}{ Occurrences } & \multicolumn{3}{|c|}{ Detection tracks } & \multicolumn{2}{|c|}{ Error rates } \\
\hline & & True positives & False positives & False negatives & $f_{\mathrm{F}}: \%$ & $f_{\mathrm{N}}: \%$ \\
\hline Balise & 132 & 132 & 15 & 0 & 10.20 & 0 \\
\hline PZB inductor & 150 & 150 & 9 & 0 & 5.66 & 0 \\
\hline Signal & 118 & 118 & 23 & 0 & 16.31 & 0 \\
\hline Signal gentry & 11 & 11 & 15 & 0 & 57.69 & 0 \\
\hline Railroad switch & 103 & 103 & 35 & 0 & 25.36 & 0 \\
\hline Panel & 203 & 199 & 125 & 4 & 38.58 & 1.97 \\
\hline Axle counter & 133 & 124 & 2 & 9 & 1.59 & 6.77 \\
\hline Railway platform & 24 & 22 & 28 & 2 & 56.00 & 8.33 \\
\hline Fouling point & 93 & 79 & 4 & 14 & 4.82 & 15.05 \\
\hline Total & 967 & 938 & 256 & 29 & 21.44 & 3.00 \\
\hline
\end{tabular}

The results are based on a pool of different on-site measurement videos from Germany, with a total duration of more than $3 \mathrm{~h}$

suitable for image recognition, and their use has been successfully tested for symbol recognition purposes by Stoitchkov (2018).

The symbols that need to be recognised (Figure 9) are rather small compared with the complete drawing and do not occur in large numbers. Also, on most technical drawings, there is much white space where it is unreasonable to scan for a symbol, as nothing is depicted. Therefore, it makes sense to divide the drawing into rectangular sections - that is, ROIs - and to scan only those ROIs that are not empty. In other words, ROIs that contain white pixels only are ignored in order to minimise the amount of data and thereby the computational effort.

The size of those ROIs is a critical parameter, as the computational effort decreases disproportionally when very small ROIs are used. In the case of large ROIs, the training time increases drastically while at the same time the detection accuracy decreases. It is also crucial how the drawing is divided into ROIs. It is possible that a significant symbol is located on a border between two ROIs and thus will not be detected, as the partial symbol cannot be recognised by the CNN in either of the two ROIs. The authors found that the simplest solution for this problem is an overlap of the ROIs at the expense of some computational overhead.

\subsubsection{Generation of training data}

Providing thousands of input images for the training process is usually a big challenge and can result in tedious work if the images have to be selected and classified by hand. In the case of symbol detection, training images can be generated artificially due
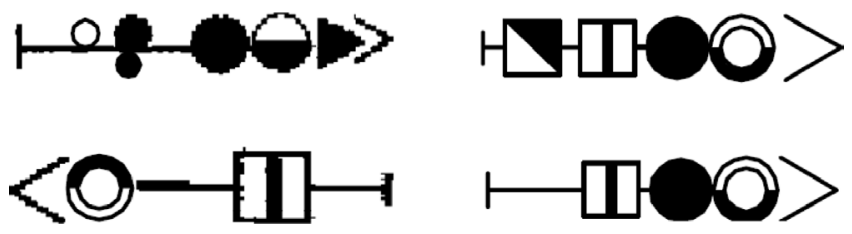

Figure 9. Samples of symbols representing different types of railway signals that the network was trained to detect to the inherently repetitive nature of drawing symbols. This generation process is performed in the following steps.

In the first step, a large background image was created by grouping a number of random image parts together, such as parts of technical drawings, text elements and random patterns. Subsequently, thousands of smaller images, containing the symbol to be detected, were created. These images are used for training the CNN and have the same size as the ROIs in the recognition process. These images were created by copying random parts of the same size from the background image and placing the symbol onto each of them. To ensure that a large variety of images was generated, this process was entirely randomised, through the selection of arbitrary parameters, such as the position and rotation of the symbol relative to the image (see Figure 10).

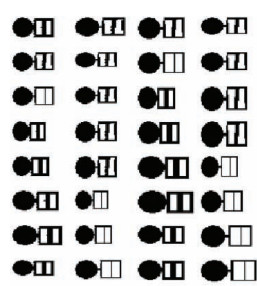

Symbol masks

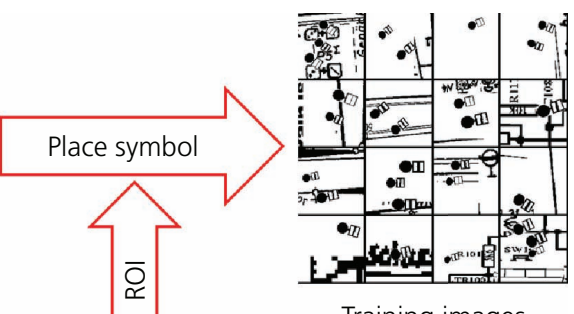

Training images

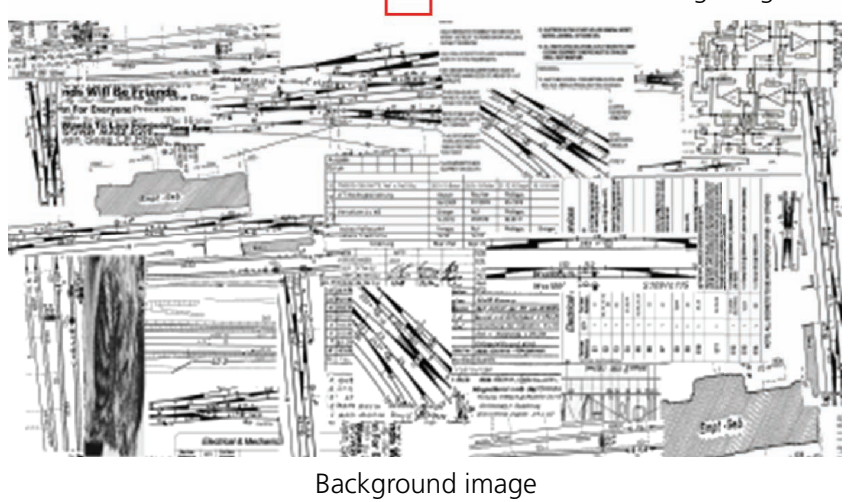

Figure 10. Cutting the background image into ROls and placing training symbols in different configurations 
However, intensive testing did not yield sufficiently accurate prediction results (on average, there were 13 false positives per single drawing). The main reason for the inaccurate detection is the similarity between different symbols in the drawings, which are very difficult to distinguish from each other. Finding a particular symbol often leads to other similar symbols being recognised, which is not the goal. Therefore, it was concluded that an improvement of the generation process was necessary. A first attempt adapted the background image such that it mostly contained elements such as line shapes, numbers and text that may occur in the technical drawings to which the presented method is applied. The random patterns put into the background image in advance did not result in any increase in training accuracy. The random elements (e.g. line shapes and letters that appear on drawings but are not the symbols to be detected), however, helped reduce the detection of false positives. After adjusting the background image, the detection of false positives was drastically reduced. The number of average false positives per drawing was reduced from 13 with a standard background down to 0.5 with the adjusted background. Afterwards, a set of variations of the symbol is created (the symbol masks in Figure 10), which was achieved by resizing and stretching the symbol. Each of these symbol variations is placed randomly in different ROIs for the training process. These measures increase the detection capabilities of the CNNs and therefore reduce the number of false negatives.

\subsection{Training process}

Before the training process can be initiated, it is necessary to compile a CNN model. To create this $\mathrm{CNN}$, model various parameters have to be adjusted - for example, the number and type of layers, the number of filter types and the type of activation function (see Section 2.3). As there is no single perfect solution for setting up the model, the best parameters had to be determined by way of systematic experimentation. In this regard, different model set-ups were created by changing one parameter per set-up and training and testing them for accuracy. The models with the best results were then further refined until a model set-up with excellent recognition capability was obtained. In each of these refinement stages, the training was executed with only a reduced number of input images (a few hundred), as this process otherwise would have been inappropriately time consuming.

After that, a CNN with the obtained model set-up and a few thousand input images was trained on and applied to real technical drawings of railway lines. The results of these scans revealed that the model still could not guarantee a detection rate of more than $90 \%$, as it failed to detect symbols that are located close to each other.

To increase further the detection accuracy, the CNN was split into three more specialised CNNs. The first CNN is trained only to recognise how many of the specific symbols are present in an ROI. If no symbol is detected, the program skips to the next ROI and checks again. If the first $\mathrm{CNN}$ has detected a single symbol, the second $\mathrm{CNN}$ (CNN for one symbol) is used to determine the exact position of the symbol in the ROI. Alternatively, if two symbols are found, the second CNN (CNN for two symbols) is used to determine the positions of both symbols (see Figure 11). The size of the ROIs and the overlap between them are chosen so that in almost all cases there are no more than two symbols in a single ROI, so no scanning is performed for more than two symbols.

\subsubsection{Overfitting}

Because the training images are generated artificially, there is a high chance of overfitting (see Section 2.3). Therefore, two types of input symbols are required. The first type (the training symbol) is a set of up to five images depicting different variants of the symbol that needs to be detected. With these images, the training data set was generated. The other testing symbol is cut out from a real - and presumably slightly distorted - drawing and used to generate another set, also with a different background image - the testing data set. From the training data set, $15 \%$ are taken as validation data, which are also used for calculating the accuracy. The accuracy for both the validation and testing data set is measured, and the average is taken as the final accuracy.

The training process is as follows: train one epoch, apply the trained neural network on both image sets, measure the accuracy and store the neural network only if it yields the best accuracy compared with the prior epochs. If the neural network overfits over the epochs, the accuracy of the test data will decrease, and because of the averaging with the training data set, the overfitted network will be discarded.

\subsection{Implementation and results of the training process \\ 4.3.1 Facilitating the training and recognition process}

The authors combined the developed algorithms with a graphical user interface (GUI) in order to simplify their usage and to allow the user to flag false positives. This information can be used for improving the training process of new symbols.

The GUI allows drawings of several formats to be imported, such as PDF, PNG or TIF, and the detection of pretrained symbols in the imported drawings. The positions of the symbols are then visualised and can be exported as text files or as annotations at the positions of

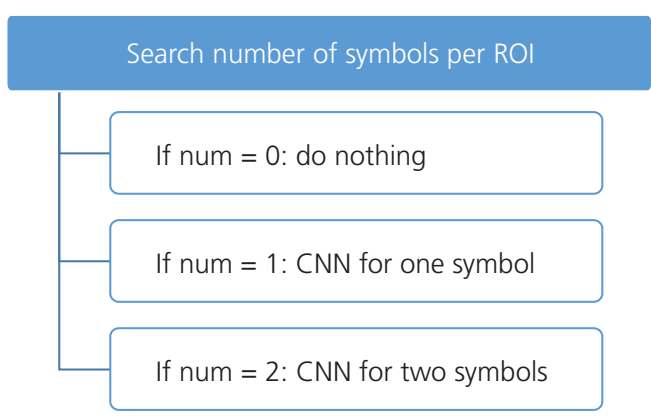

Figure 11. First, it is determined how many symbols are in one ROI. Depending on the outcome, either the ROI is skipped or a specific CNN activated in order to locate the symbol(s) 
Smart Infrastructure and Construction

Volume 172 Issue 1
Recognising railway infrastructure

elements in videos and drawings using

neural networks

Vilgertshofer, Stoitchkov, Borrmann, Menter and Genc detected symbols in PDF files. Another feature is the option of training the necessary CNNs automatically for new symbols. This can be done by using the predefined parameters or using advanced options that control, for example, the ROI size or number of epochs.

Provided that the user has already trained at least one set of $\mathrm{CNNs}$, the workflow of the software is straightforward. The technical drawings that should be evaluated are imported and displayed for the user to check if any mistakes occurred in the conversion process. After that, the drawing is analysed to detect one or various symbols by using the respective set of CNNs. The results of this detection are then presented on the same screen as a list of the found symbols and their position in pixel coordinates. All results can be selected one by one, and on selection, their position on the drawing is visualised by cross-hairs as well as a zoomed image. At this stage, the user can manually delete falsely detected symbols and check the overall quality of the scan. Finally, the user can export the obtained and reviewed results. Figure 12 shows the workflow schematically.

\subsubsection{Used software}

Keras, which runs on top of TensorFlow (Abadi et al., 2016), was used to build and train the CNNs. the authors avoided conflicts with the compatibility of different programming languages by writing the GUI with the TkInter library, which is the standard GUI in Python. The CNN architecture has been specifically designed to provide the highest accuracy while minimising overfitting. No pretrained neural networks have been used because the symbols are very simple structures and the common pretrained $\mathrm{CNNs}$ do not contribute to accuracy in this case. The architecture of the CNN is relatively simple. It consists of five convolution layers with a kernel size of $4 \times 4$, between each of which there is a max pooling layer with a pool size of $2 \times 2$. Thereafter, there are four densely connected layers with a dropout layer of 0.2 between each for preventing overfitting (see Section 2.3).

\subsubsection{Accuracy}

The accuracy is measured in two different ways. For the number of symbols, it is measured in per cent, where the recognised number is compared with the ground truth number of symbols in the ROI. On the other hand, the accuracy for the location of a symbol is measured as a distance in pixels, where the distance denotes the deviation from the recognised coordinates to the

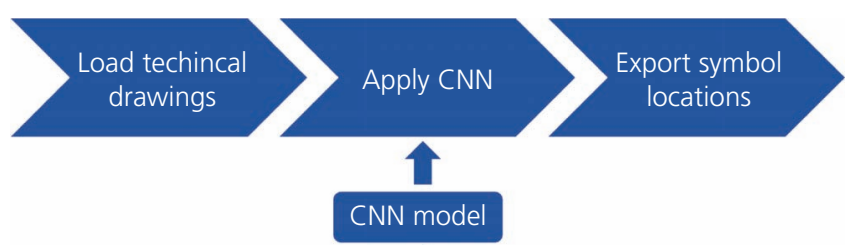

Automatic training Automatic scanning

Figure 12. NNdips workflow ground truth coordinates of a given symbol. The accuracy of the network develops in the course of the training process, which is performed in epochs as described in Section 2.3.

Figure 13 shows the accuracy of the detected number of symbols, and it can be seen that the network learns very fast for this specific task. Figure 14 shows the learning curve for the location of one symbol. Here, the learning happens more slowly, and more time is required for accurate results. The authors explain this as

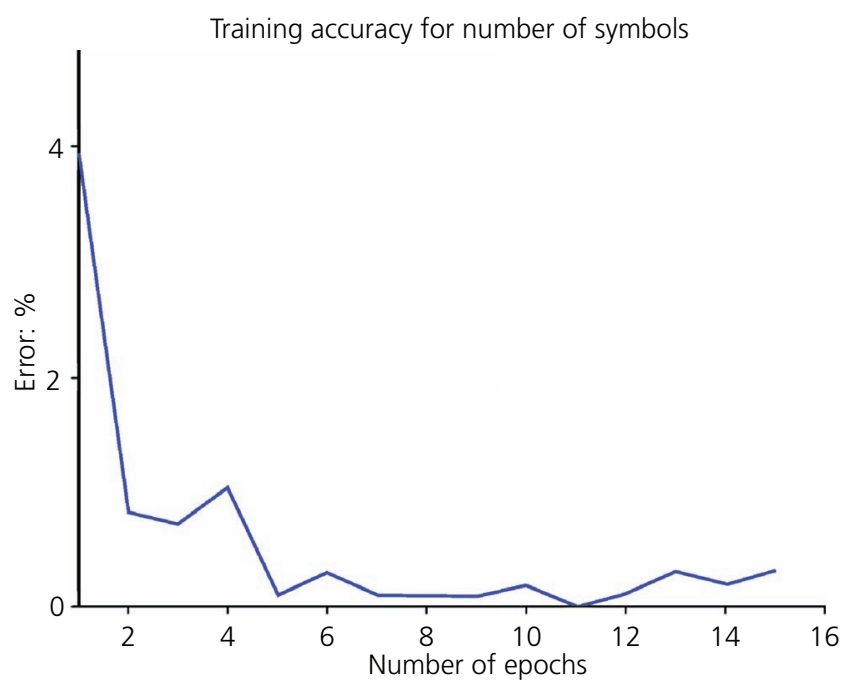

Figure 13. Number of symbols per ROI: development of the network accuracy during the course of the training process (epochs). The $y$-axis shows the percentage of ROls in which the number of symbols was not correctly recognised

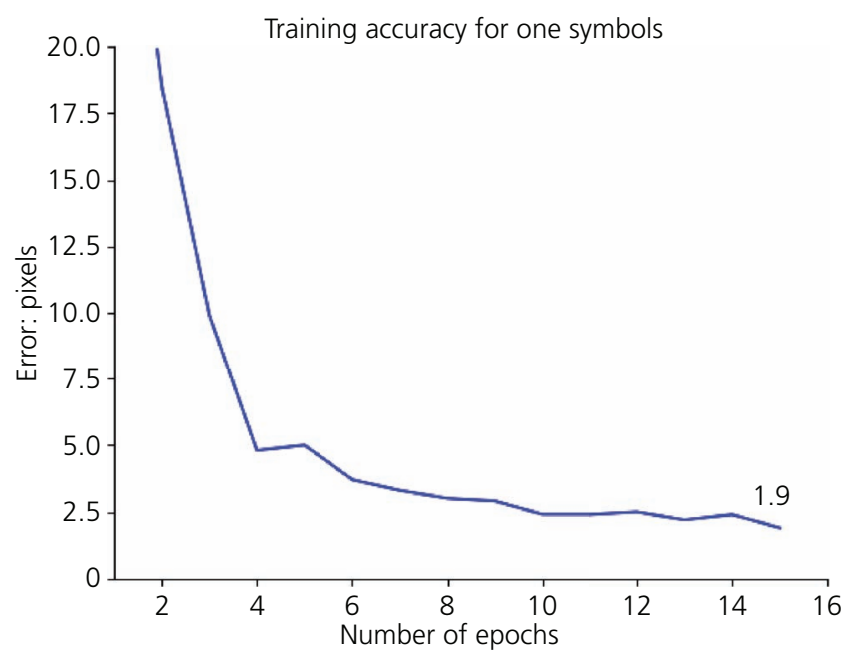

Figure 14. Location of one symbol per ROI: development of the network accuracy during the course of the training process (epochs). The $y$-axis shows the error in terms of the average deviation in pixels from the correct position of the symbol in the image 
Table 2. Results of the verification process

\begin{tabular}{|c|c|c|c|c|c|c|}
\hline Symbol & $\begin{array}{l}\text { Manual count } \\
\text { (ground truth) }\end{array}$ & $\begin{array}{l}\text { Symbols found } \\
\text { by NNdips }\end{array}$ & $\begin{array}{l}\text { NNdips without } \\
\text { double detections }\end{array}$ & $\begin{array}{l}\text { False } \\
\text { positives }\end{array}$ & $\begin{array}{l}\text { False } \\
\text { negatives }\end{array}$ & $\begin{array}{c}\text { False } \\
\text { negatives: \% }\end{array}$ \\
\hline$\infty$ & 215 & 366 & 197 & 9 & 27 & 12.56 \\
\hline 9्रा & 4 & 7 & 4 & 0 & 0 & 0 \\
\hline ๑ा & 22 & 55 & 35 & 16 & 3 & 13.64 \\
\hline ए: & 13 & 42 & 37 & 25 & 1 & 7.69 \\
\hline 获 & 37 & 70 & 34 & 2 & 5 & 13.51 \\
\hline 60 & 28 & 51 & 30 & 2 & 0 & 0 \\
\hline 68 & 16 & 24 & 14 & 0 & 2 & 12.50 \\
\hline$\Lambda$ & 96 & 156 & 90 & 0 & 6 & 6.25 \\
\hline$\otimes$ & 20 & 43 & 21 & 1 & 0 & 0 \\
\hline (4) & 29 & 74 & 49 & 20 & 0 & 0 \\
\hline 0 & 43 & 133 & 76 & 36 & 3 & 6.98 \\
\hline
\end{tabular}

being due to the complexity of the problem. Deciding between the number of symbols (three possible results: 0,1 or 2) in an ROI is an easier task than to find the exact $x$ and $y$ coordinates of a symbol in an ROI. Both figures were created in the scope of the training process with the default values of the ROI size $(160 \times$ 160 pixels) for 15 epochs and 23996 training images, 4235 validation images and 4028 testing images. When tested on different symbols, the accuracy was consistently above $99 \%$ in terms of recognising how many symbols are in a single ROI. Furthermore, the trained CNNs showed on average less than 2 pixel deviation for the location of the symbol. However, when the neural network is applied to a drawing, there are thousands of ROIs to be searched. As there is a possible error of between 0 and $1 \%$ in each ROI, the overall error multiplies with the number of ROIs as can be seen in Table 2 .

\subsection{Proof of concept and use-case scenario}

The output of the CNNs is the coordinates for each found symbol. These can be visualised by marking the position in the associated drawing. Figure 15 shows an example of the output in a PDF file. The positions are marked as annotations. Another possibility is the export of a TXT file. It is necessary for users to supervise the outputs in order to flag false positives and to comment on single results in the GUI - for example, to add the kilometrage to a symbol.

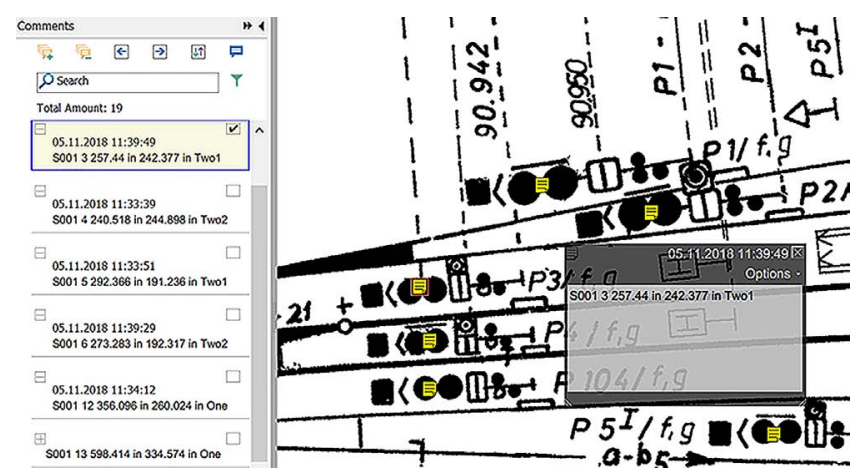

Figure 15. Visualisation of the results in a PDF file. The position of the recognised symbols is marked in yellow
So far, the networks have been tested with over 25 different drawings provided by DB Netz AG. In those drawings, more than ten symbols were selected to verify the previously described method. Table 2 summarises the results of this verification process. Each line shows the actual number of symbols present in a set of drawings (column manual count). To guarantee that this number is accurate, manual counting was conducted by three different persons. The column symbols found by NNdips shows the number of hits that the CNN produced. Since it is usual for multiple hits for the same symbol to occur, the number in the column 'NNdips without double detections' shows the actual number of symbols found - without counting the multiple hits that can be removed in post-processing. In some cases, the CNN produced a false-positive result (a hit at a position where no symbol is present) - the respective number is given in the column 'False positives'. Unfortunately, the CNN does not recognise a symbol at all times. The count of these false negatives (symbols missed by the $\mathrm{CNN}$ ) is shown in the column 'False negatives' and given as a percentage value in the last column (the percentage is calculated by dividing the number of false negatives by the number of actually present symbols e.g. $27 / 215=12.56$ in the first row).

While those results are not perfect, they nonetheless indicate that the approach shows promising possibilities - particularly in regard to the very short time required for training the CNNs (see below).

\subsubsection{Challenges}

Training CNNs for the detection of symbols in technical drawings poses multiple challenges. Previous knowledge about the general occurrence of the symbols is essential. In the given technical drawings, the occurrence of multiple symbols close together was noted in several occasions. This results in a multiple detection of the same symbol in different ROIs. This is a well-known issue, particularly in object detection in videos (see Section 3.1).

Other works (Breuers et al., 2016) tackle this by deleting multiple hits by calculating an overlap-area ratio of bounding boxes around the detected objects. The authors did not apply any of the possible techniques here, as the drawing data yield cases where multiple symbols are incredibly close together, and a minimum-distance approach or a bounding-box overlap could delete distinct hits. 
Further investigation of this phenomenon could minimise the number of multiple detections of symbols.

Another challenge is the rare case of more than two symbols in a single ROI that are not detected by the overlap of ROIs. The results of tests with an additional CNN to detect the positions of three (instead of only one or two) symbols in one ROI, however, lacked accuracy. The CNN substantially increased the number of false positives, as the same symbol was detected multiple times. If in further testing, symbols are missed by the algorithm due to more than two symbols in an ROI, this error can presumably be corrected by merely reducing the ROI size. Additional testing would be required to prove this assumption.

\subsubsection{Computing time for training and recognition}

When using the parameters and symbols shown in this paper, the time for the training process of a single symbol took between 20 and $30 \mathrm{~min}$ on a computer with an eight-core i7-7700 central processing unit at $3.60 \mathrm{GHz}$ and an Nvidia Quadro P2000 graphics card. The recognition process, which consists of the calling of the trained model and highlighting of the symbols on a single drawing, took about $10 \mathrm{~s}$ on the same machine. Similar results could be achieved on a laptop with an Nvidia GeForce 940MX graphics card; thus, the training can potentially be done within a reasonable time on any modern graphics card.

\section{Combination of the results and data fusion}

The preceding Sections 3 and 4 have introduced two complementary parts of an overall approach with the goal of supporting the capture, analysis and consistency checking of railway -from both real-world data and technical drawings. Both parts were combined to enable this semi-automated consistency checking of technical drawings and the corresponding capture railway infrastructure. For this purpose, video and data from drawings for a specific stretch of a railway line were collected, analysed and compared. Two example results of this process are shown in Figures 16 and 17.

To achieve this, the results of both parts were further processed in order to make them comparable. From the results of analysing the video data, the location - that is, the kilometrage - and the type of a detected element can be exported. This is possible as the location of the train is tracked during the recording of each railway track video file. This allows linking each frame to a specific kilometrage. However, this process is not described in detail, as it is not the focus of this paper and has been practically applied by the Signon Deutschland $\mathrm{GmbH}$ for many years. The results of analysing the technical drawings are also post-processed to export the kilometrage of the detected symbols. As the main focus was the automatic detection of specific symbols in large drawings containing many different symbols, the corresponding recognition of the kilometrage was not automated. Therefore, each located symbol has to be annotated manually with the kilometrage next to the symbol on the drawing (see Figure 17 for an example of the kilometrage given on a drawing). Thereby, the kilometrage and the type of a detected element are exported in the same format as the results of analysing the video data. Both lists of symbol types and locations are then compared to check the consistency - for example, the number of symbols and if a certain symbol is located at the same kilometrage in both data sets. In general, the combination of both methods yields many advantages if compared with applying only one method and particularly if compared with a manual capturing process.

- Analysis of the video data of railway lines with a machinelearning-based method is very fast compared with a manual

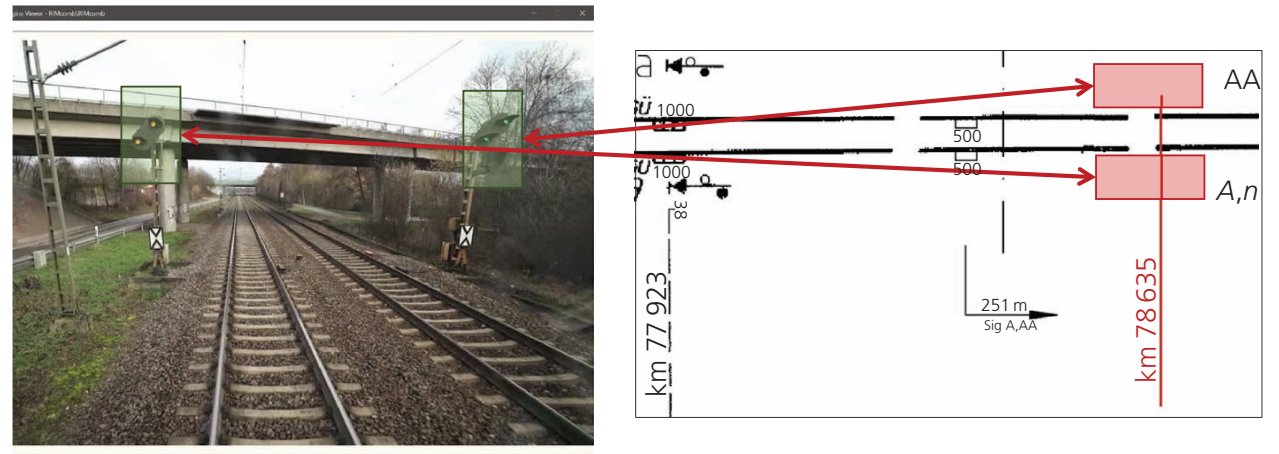

\begin{tabular}{lccc}
\multicolumn{2}{c}{ Manual checking } & \multicolumn{2}{c}{ Automatic checking } \\
Video & Drawing & Drawing (NNdips) & Video (RIMcognize) \\
\hline $78635 \mathrm{~km}$ & - & - & $78635.0 \mathrm{~km}$ \\
$78635 \mathrm{~km}$ & - & - & $78635.1 \mathrm{~km}$ \\
\hline
\end{tabular}

Figure 16. Comparison of the results of RIMcognize and NNdips. Here, an inconsistency was detected, since elements were recognised in the video data but not in the corresponding drawings 


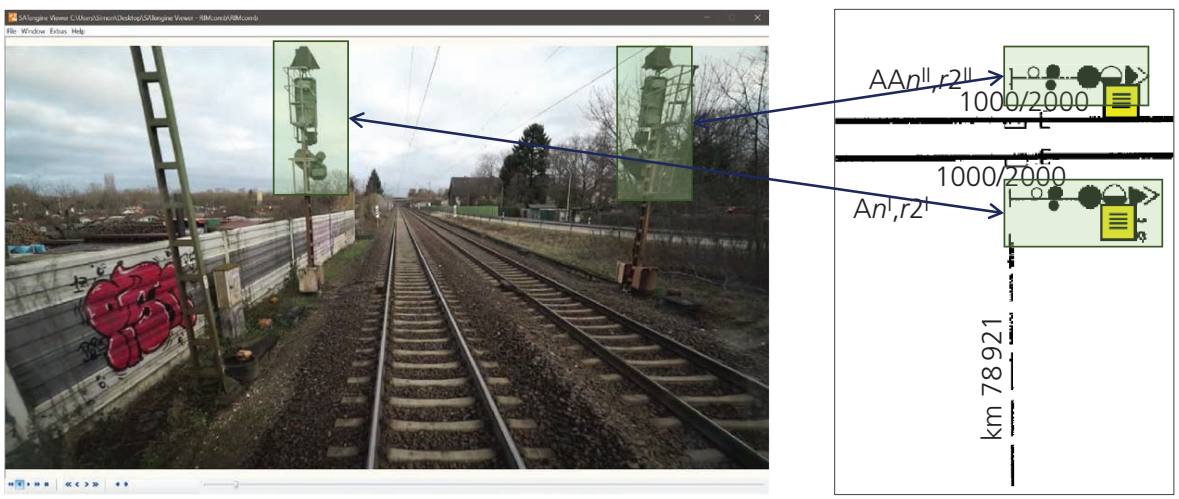

\begin{tabular}{lccc}
\multicolumn{2}{c}{ Manual checking } & \multicolumn{2}{c}{ Automatic checking } \\
Video & Drawing & Drawing (NNdips) & Video (RIMcognize) \\
\hline $78921 \mathrm{~km}$ & $78921 \mathrm{~km}$ & $78921 \mathrm{~km}$ & $78921.3 \mathrm{~km}$ \\
$78921 \mathrm{~km}$ & $78921 \mathrm{~km}$ & $78921 \mathrm{~km}$ & $78921.4 \mathrm{~km}$
\end{tabular}

Figure 17. Comparison of the results of RIMcognize and NNdips. In this case, the built situation is sufficiently consistent with the details displayed in the corresponding drawing

process. While elements might be missed by the computer, this is also the case for a human performing this task.

- The labour-intensive task of analysing large technical drawings in order to locate specified plan symbols can also be supported by machine learning, as the symbols are directly highlighted and can be further described by a user who adds the kilometrage. When combining the results of both methods, it can be checked if a drawing is up to date: are there missing elements on a drawing or are elements depicted that do not exist on the real railway line?

- Another benefit of the combination is that false negatives in the video can be identified if the element is detected on a corresponding drawing. The possibility of having a false negative in the analysis of the video and in the analysis of the drawing is lower compared with that of applying only one method.

v As each method can verify the results of the other method, both methods can symbiotically improve one another.

However, human checking and quality control is always necessary to verify the results of the method.

\section{Conclusion and outlook}

In this paper, a method is introduced that contributes to the validation of the real-world situation against representations on technical drawings in the field of railway infrastructure. One part of the approach automatically recognises important symbols in technical drawings by using a CNN.

It is shown how the approach was developed, implemented and tested in order to reduce the manual effort that would otherwise be necessary in order to obtain digital information from the aforementioned technical drawings. At this stage, the accuracy of the method is only approximately $90 \%$ (depending on the symbol, up to
$10 \%$ of the instances in a drawing are not detected by the CNN, i.e. false negatives). Therefore, the automated training process is under further development in order to improve the accuracy for all types of symbols. Nonetheless, the presented training and detection process has various advantages when considering that manual checking by a human is an error-prone task. It does not require a large number of manually labelled images, and it is both automated and fast. Therefore, it can be concluded that the discussed approach helps in reducing the high manual effort required for checking the accuracy of technical drawings of railway infrastructure and provides significant support for building up a digital twin of railway infrastructure.

This information can then be further used as the basis for maintaining or planning any refurbishment and reconstruction of railway infrastructure. It can also support the task of checking the accuracy of technical drawings against the actual as-built situation. The as-built situation can automatically be detected with the part of the approach described in Section 3 of this paper. Here, video data from railway lines are analysed using a trackingby-detection approach, which automatically recognises various infrastructure elements. This second approach also uses CNNs for the detection of elements by analysing single frames in the video.

Both methods were combined to enable a semi-automated consistency check of technical drawings. For this purpose, video data, as well as plan data for a specific stretch of railway track, were collected, analysed and compared.

In future work, this consistency checking will be further automated. To date, the number next to a symbol that was automatically detected has been manually extracted from the drawing to enable comparison. This number is called the 'kilometrage' and identifies the location of 
the particular infrastructure element. While recognising the letters or numbers in an image is well developed, further research is necessary to assign automatically the correct kilometrage to a specific element, particularly in situations where many symbols, texts and numbers occur close to each other.

\section{Acknowledgement}

The authors gratefully acknowledge the support of the Bavarian Research Foundation in funding the project.

\section{REFERENCES}

Abadi M, Agarwal A, Barham P et al. (2016) TensorFlow: Large-scale Machine Learning on Heterogeneous Distributed Systems. arXiV.org, Cornell University, Ithaca, New York, NY, USA. See http://arxiv.org/ abs/1603.04467 (accessed 04/07/2020)

Benenson R, Omran M, Hosang J and Schiele B (2014) Ten years of pedestrian detection, what have we learned? In Computer Vision ECCV 2014 Workshops (Agapito L, Bronstein M and Rother C (eds)). Springer, Cham, Switzerland, pp. 613-627.

Bergmann P, Meinhardt T and Leal-Taixe L (2019) Tracking without Bells and Whistles. arXiV.org, Cornell University, Ithaca, New York, NY, USA. See http://arxiv.org/abs/1903.05625 (accessed 04/07/2020).

Bewley A, Ge Z, Ott L, Ramos F and Upcroft B (2016) Simple Online and Realtime Tracking. arXiV.org, Cornell University, Ithaca, New York, NY, USA. See http://arxiv.org/abs/1602.00763 (accessed 04/07/2020).

Bochinski E, Eiselein V and Sikora T (2017) High-speed tracking-bydetection without using image information. Proceedings of 2017 14th IEEE International Conference on Advanced Video and Signal Based Surveillance (AVSS), Lecce, Italy, pp. 1-6.

Bochinski E, Senst T and Sikora T (2018) Extending IOU based multiobject tracking by visual information. Proceedings of the 2018 15th IEEE International Conference on Advanced Video and Signal Based Surveillance, Auckland, New Zealand, pp. 441-446.

Breuers S, Yang S, Mathias M and Leibe B (2016) Exploring bounding box context for multi-object tracker fusion. Proceedings of 2016 IEEE Winter Conference on Applications of Computer Vision (WACV), Lake Placid, NY, USA, pp. 1-8.

Dollár P, Appel R, Belongie SJ and Perona P (2014) Fast feature pyramids for object detection. IEEE Transactions on Pattern Analysis and Machine Intelligence 36(8): 1532-1545, https://doi.org/10.1109/ TPAMI.2014.2300479.

Felzenszwalb PF, Girshick RB, McAllester D and Ramanan D (2010) Object detection with discriminatively trained part-based models. IEEE Transactions on Pattern Analysis and Machine Intelligence 32(9): 1627-1645, https://doi.org/10.1109/TPAMI.2009.167.

Genc C, Harmsen F and Köhler T (2018) Automated recognition of infrastructure elements using neural networks. SIGNAL+DRAHT 110(9): 48-55.

Girshick R, Donahue J, Darrell T and Malik J (2014) Rich feature hierarchies for accurate object detection and semantic segmentation. Proceedings of the 2014 IEEE Conference on Computer Vision and Pattern Recognition, Columbus, OH, USA, pp. 580-587.

He K, Zhang X, Ren S and Sun J (2015) Deep Residual Learning for Image Recognition. arXiV.org, Cornell University, Ithaca, New York, NY, USA. See http://arxiv.org/abs/1512.03385 (accessed 04/07/2020).

Huang J, Rathod V, Sun C et al. (2016) Speed/Accuracy Trade-offs for Modern Convolutional Object Detectors. arXiV.org, Cornell University, Ithaca, New York, NY, USA. See http://arxiv.org/abs/1611 10012 (accessed 04/07/2020).

Le Bodic P, Héroux P, Adam S and Lecourtier Y (2012) An integer linear program for substitution-tolerant subgraph isomorphism and its use for symbol spotting in technical drawings. Pattern Recognition 45(12): 4214-4224, https://doi.org/10.1016/j.patcog.2012.05.022.
LeCun Y, Bottou L, Bengio Y and Haffner P (1998) Gradient-based learning applied to document recognition. Proceedings of the IEEE 86(11): 2278-2324, https://doi.org/10.1109/5.726791.

Luqman MM, Brouard T and Ramel JY (2009) Graphic symbol recognition using graph based signature and Bayesian network classifier. Proceedings of 2009 10th International Conference on Document Analysis and Recognition, Barcelona, Spain, pp. 1325-1329.

Nayef N, Afzal MZ and Breuel TM (2012) Learning feature weights of symbols, with application to symbol spotting. Proceedings of the 21st International Conference on Pattern Recognition (ICPR2012), Tsukuba, Japan, pp. 2371-2374.

Papageorgiou C and Poggio T (2000) A trainable system for object detection. International Journal of Computer Vision 38(1): 15-33, https://doi.org/10.1023/A:1008162616689.

Ren S, He K, Girshick R and Sun J (2015) Faster R-CNN: towards real-time object detection with region proposal networks. In Advances in Neural Information Processing Systems 28 (Cortes C, Lawrence ND, Lee DD, Sugiyama M and Garnett R (eds)). Curran Associates, Red Hook, NY, USA, pp. 91-99.

Rezvanifar A, Cote M and Albu AB (2019) Symbol spotting for architectural drawings: state-of-the-art and new industry-driven developments. IPSJ Transactions on Computer Vision and Applications 11(1): article 2, https://doi.org/10.1186/s41074-019-0055-1.

Rezvanifar A, Cote M and Branzan Albu A (2020) Symbol spotting on digital architectural floor plans using a deep learning-based framework. Proceedings of the 2020 IEEE/CVF Conference on Computer Vision and Pattern Recognition (CVPR) Workshops, Seattle, WA, USA, pp. 568-569.

Rusiñol M and Lladós J (2005) Symbol spotting in technical drawings using vectorial signatures. In Graphics Recognition: Ten Years Review and Future Perspectives (Liu W and Lladós J (eds)). Springer, Berlin, Germany, pp. 35-46.

Stoitchkov D (2018) Analysis of Methods for Automated Symbol Recognition in Technical Drawings. Bachelor's thesis, Technical University of Munich, Munich, Germany.

Stoitchkov D, Breier P, Slepicka M et al. (2019) Automatic detection of plan symbols in railway equipment engineering using a machine learning approach. Proceedings of the 2019 European Conference on Computing in Construction, Chania, Crete, Greece, pp. 92-99.

Süße H and Rodner E (2014) Bildverarbeitung und Objekterkennung: Computer Vision in Industrie und Medizin. Springer, Wiesbaden, Germany (in German). See https://books.google.de/books? id=YDBwBAAAQBAJ (accessed 04/07/2020).

Szegedy C, Vanhoucke V, loffe S, Shlens J and Wojna Z (2015) Rethinking the Inception Architecture for Computer Vision. arXiV.org, Cornell University, Ithaca, New York, NY, USA. See http://arxiv.org/abs/1512. 00567 (accessed 04/07/2020).

Talukdar J, Gupta S, Rajpura PS and Hegde RS (2018) Transfer learning for object detection using state-of-the-art deep neural networks. Proceedings of the 2018 5th International Conference on Signal Processing and Integrated Networks (SPIN), Noida, India, pp. 78-83.

Vilgertshofer S, Stoitchkov D, Esser S et al. (2018) The RIMcomb research project: towards the application of building information modeling in railway equipment engineering. In eWork and eBusiness in Architecture, Engineering and Construction (Karlshoj J and Scherer R (eds)). CRC Press, London, UK, pp. 439-445.

Viola P and Jones M (2004) Robust real-time object detection. International Journal of Computer Vision 57(2): 137-154, https://doi. org/10.1023/B:VISI.0000013087.49260.fb.

Weber J and Tabbone S (2012) Symbol spotting for technical documents: an efficient template-matching approach. Proceedings of the 21st International Conference on Pattern Recognition (ICPR 2012), Tsukuba, Japan, pp. 669-672.

Wojke N, Bewley A and Paulus D (2017) Simple Online and Realtime Tracking with a Deep Association Metric. arXiV.org, Cornell 
Smart Infrastructure and Construction Volume 172 Issue 1
Recognising railway infrastructure

elements in videos and drawings using

neural networks

Vilgertshofer, Stoitchkov, Borrmann, Menter and Genc
University, Ithaca, New York, NY, USA. See http://arxiv.org/abs/1703. 07402 (accessed 04/07/2020).

Wu Y, Lim J and Yang M (2013) Online object tracking: a benchmark. Proceedings of the 2013 IEEE Conference on Computer Vision and Pattern Recognition, Portland, OR, USA, pp. 2411-2418.

Yoon Y, Kim DY, Yoon K, Song Y and Jeon M (2019) Online Multiple Pedestrian Tracking Using Deep Temporal Appearance
Matching Association. arXiV.org, Cornell University, Ithaca, New York, NY, USA. See http://arxiv.org/abs/1907.00831 (accessed 04/07/2020).

Ziran Z and Marinai S (2018) Object detection in floor plan images. In Artificial Neural Networks in Pattern Recognition (Pancioni L, Schwenker F and Trentin E (eds)). Springer, Cham, Switzerland, pp. 383-394.

\section{How can you contribute?}

To discuss this paper, please email up to 500 words to the editor at journals@ice.org.uk. Your contribution will be forwarded to the author(s) for a reply and, if considered appropriate by the editorial board, it will be published as discussion in a future issue of the journal.

Proceedings journals rely entirely on contributions from the civil engineering profession (and allied disciplines). Information about how to submit your paper online is available at www.icevirtuallibrary.com/page/authors, where you will also find detailed author guidelines. 